Rev. Elev. Med vét. Pays trop., 1975, 28 (4) : 491-497

\title{
Note sur les facteurs conduisant au choix d'une saison de monte au C.R.Z. de Dara (Sénégal)
}

\author{
par J. P. DENIS $\left({ }^{*}\right)$ et A. I. THIONGANE (**)
}

\begin{abstract}
RESUME
Afin de déterminer la période la plus favorable pour installer une saison de monte chez des zébus Gobra de la zone Nord du Sénégal, l'étude de la répartition naturelle des naissances, de la fréquence mensuelle des saillies, de l'âge au 1 $1^{\text {er }}$ vêlage, de l'intervalle entre les vêlages en fonction du mois de naissance du produit, de l'évolution pondérale en fonction de l'époque de naissance chez les femelles et les produits, de la mortalité, de la commodité de gestion du troupeau, de la supplémentation alimentaire, a été abordée. Il en résulte que cette période de monte doit se situer de la mi-août à la fin novembre pour la région considérée.
\end{abstract}

\section{INTRODUCTION}

En zone sahélienne, l'existence de conditions climatiques extrêmement variables au cours de l'année a pour conséquence des variations saisonnières importantes des différents facteurs de la vie du troupeau: alimentation, reproduction, physiologie, évolution pondérale des jeunes et des adultes.

Les phénomènes qui touchent la fonction de reproduction sont les plus importants. Ils conditionnent directement le but essentiel, c'est-àdire l'obtention du maximum de produits dont la croissance se déroulera dans les meilleures conditions possibles. Une saison de monte dort être instituée donc pour les raisons suivantes :

- Etant donné les fortes variations des conditions d'environnement durant l'année, il y a intérêt à regrouper toutes les naissances à une même période pour faciliter les com-

(*) Docteur vétérinaire I.E.M.V.T., Service de Zootechnie du Laboratoire national de l'Elevage et de Recherches vétérinaires, B.P. 2057, DakarHann (Sénégal).

(**) Docteur vétérinaire, Directeur du Centre de Recherches Zootechniques de Dara-Djoloff (Sénégal). paraisons de croissances des produits. Ceci est surtout valable en station de sélection;

- Il y a intérêt à regrouper aussi les naissances pour faciliter les différentes opérations nécessaires à la conduite de l'élevage: sevrage, vaccinations, supplémentation en saison sèche, mise à la reproduction, testage des taureaux.

Le présent travail contient des données et des remarques exposées dans divers travaux précédents concernant le zébu Gobra et un certain nombre de résultats originaux. Il s'agit ici de regrouper dans une même étude tout ce qui peut concerner la mise en application dune saison de monte.

Dans une première partie, les facteurs entrant en jeu dans le choix de cette saison seront donnés. Dans la seconde, la valeur de chacun des facteurs étudiés sera discutée, puis les facteurs déterminants conduiront à la mise en évidence de l'époque la plus favorable pour la monte.

Enfin, quelques résultats de cette opération seront donnés après quatre années de mise en pratique. 


\section{FACTEURS ENTRANT EN JEU DANS LE CHOIX}

\section{Répartition naturelle des naissances $(6,7)$}

Jusqu'en 1969, au C.R.Z. de Dara, les taureaux ont été laissés en permanence dans les différents troupeaux de femelles. Dans les conditions de la station qui, dans ce domaine, sont identiques à celles rencontrées à l'intérieur, l'étude de la répartition naturelle des naissances donne par conséquent une idée de la répartition naturelle des saillies. Les naissances qui ont été comptabilisées depuis 1955 ont permis d'établir le tableau $\mathrm{n}^{0}$ I qui montre que le

TABL, N'I-Répartition mensuelle des naissances de zếbus Gobra de 1955 à 1969.

\begin{tabular}{|c|c|c|}
\hline Mois & $\begin{array}{c}\text { Nombre de } \\
\text { naissances }\end{array}$ & p. 100 \\
\cline { 2 - 3 } & 153 & 5,56 \\
2 & 228 & 8,29 \\
3 & 232 & 8,43 \\
4 & 183 & 6,65 \\
5 & 321 & 11,67 \\
6 & 473 & 17,20 \\
7 & 464 & 16,87 \\
8 & 243 & 8,83 \\
9 & 159 & 5,78 \\
10 & 91 & 3,30 \\
11 & 92 & 3,34 \\
12 & 111 & 4,03 \\
\hline Total & 2750 & $100 \mathrm{p}, 100$ \\
\hline
\end{tabular}

maximum des vêlages a lieu aux mois de juin et juillet pour 34 p. 100 du total annuel des naissances. Si l'on regroupe la période allant du mois de mai au mois d'août compris, on trouve 54,4 p. 100 des naissances.

Une enquête effectuée dans le Ferlo - qui est la zone berceau du zébu Gobra - a donné des résultats similaires, le pourcentage des naissances survenues de juin à août se situe autour de 58 p. 100.

\section{Fréquence mensuelle des saillies $(6,7)$}

Dans le but de vérifier les données du tableau $n^{10} \mathrm{I}$, toutes les saillies ont été systématiquement relevées durant l'année 1968. Le nombre n'en est pas très important, mais la répartition observée au cours de l'année corrobore les résultats obtenus dans l'étude de la fréquence des naissances. Les résultats de ces observations apparaissent au tableau $\mathrm{n}^{0}$ II.

L'essentiel des saillies, soit 56 p. 100 , se produit durant les mois de septembre, octobre
TABL, $N^{0}$ II-Répartition mensuelle des saillies dans le troupeau Gobra en 1968.

\begin{tabular}{|c|c|c|}
\hline Mois & $\begin{array}{c}\text { Nombre de } \\
\text { naissances }\end{array}$ & p. 100 \\
\hline 1 & 6 & 4,87 \\
2 & 0 & - \\
3 & 1 & 0,81 \\
4 & 15 & 12,19 \\
5 & 9 & 7,31 \\
6 & 9 & 7,31 \\
7 & 5 & 4,06 \\
9 & 6 & 4,87 \\
9 & 23 & 18,69 \\
10 & 26 & 21,13 \\
11 & 20 & 16,26 \\
12 & 3 & 2,44 \\
\hline Total & 123 & $100 \mathrm{p} .100$ \\
\hline
\end{tabular}

et novembre. Cette période constitue donc une période de choix pour la monte naturelle.

Il faut noter qu'au mois d'avril existe un ressaut assez net du nombre des saillies dont la cause reste indéterminée. Cet accroissement se trouve au niveau des naissances (tableau $\mathrm{n}^{\circ}$ I) mais d'une façon assez amortie en février et mars. On peut penser d'une part à une mobilisation des réserves protéiques jusqu'alors fixées chez l'animal ou à une augmentation temporaire de la qualité du pâturage par consommation du pâturage aérien (montée de la sève et reverdissement des espèces arborées).

Age au $1^{\text {er }}$ vêlage en fonction du mois de naissance de la mère (2)

L'analyse de ce facteur montre qu'il n'existe pas de différence significative entre les différentes moyennes mensuelles et la moyenne générale et par conséquent cette donnée n'interviendra pas dans le choix de la période d'utilisation des taureaux.

Intervalles entre les vêlages en fonction du mois de naissance (1)

L'étude de l'intervalle entre les vêlages a été systématiquement entreprise. Les résultats portent sur 1254 intervalles de 534 vaches Gobra et apparaissent au tableau $n^{\circ}$ III.

Il n'existe pas de différence significative entre les mois, mais on peut noter que, dans l'ensemble, les valeurs des intervalles les plus importants se rencontrent durant la période de fin d'hivernage et post-hivernage. De toute façon, ce facteur n'aura qu'une influence réduite sur le choix de la saison de monte car le rétablissement du fonctionnement ovarien sera re- 
TABL. N"III-Intervalles entre les vêlages en fonction du mois de naissance du produit.

\begin{tabular}{|c|c|c|}
\hline Mois & Nombre & $\begin{array}{c}\text { Moyenne et intervalle } \\
\text { de conf iance }\end{array}$ \\
\hline 1 & 77 & $482,6 \pm 28,0$ \\
2 & 76 & $443,5 \pm 22,0$ \\
3 & 85 & $479,0 \pm 35,0$ \\
4 & 67 & $486,9 \pm 39,0$ \\
5 & 133 & $473,1 \pm 23,0$ \\
6 & 225 & $456,4 \pm 16,3$ \\
7 & 250 & $477,8 \pm 17,6$ \\
8 & 111 & $458,7 \pm 28,8$ \\
9 & 73 & $500,3 \pm 44,5$ \\
10 & 51 & $475,0 \pm 35,0$ \\
11 & 48 & $498,4 \pm 28,3$ \\
12 & 58 & $492,8 \pm 43,4$ \\
\hline
\end{tabular}

tardé soit par une alimentation déficiente, soit par des saillies trop précoces.

\section{Evolution pondérale en fonction de l'époque de naissance}

\section{Evolution pondérale de la mère (4)}

L'évolution pondérale des femelles en fonction du mois de naissance de leur produit a fait l'objet d'une étude particulière. En effet, il est important de savoir quelles sont les conséquences de la gestation et de la lactation sur l'état d'entretien de femelles qui sont soumises à une forte "agression » climatique.

Les observations ont été faites en utilisant la division trimestrielle de l'année soit : le premier trimestre correspondant au début de la saison sèche, le second à la fin de la saison sèche, le troisième et le quatrième au début et à la fin de l'hivernage ou post-hivernage. L'influence de la période du vêlage sur l'évolution pondérale est calculée sur deux années : évolution pondérale 1968 en fonction des vêlages 1967 et évolution pondérale 1968 en fonction des vêlages survenus la même année.

Cette étude montre que les vêlages du premier et du second trimestre sont éprouvants pour les mères car une partie de leur lactation se passe durant la saison sèche; de mềme et, a fortiori, pour les vêlages du $4^{e}$ trimestre qui entraînent une lactation qui se déroule dans son ensemble pendant la saison sèche. La conséquence en est que ce sont les femelles vêlant au $4^{e}$ trimestre qui présentent la chute de poids la plus spectaculaire au mois de juillet.

Dans le cas des vêlages du $3^{e}$ trimestre, la perte de poids des animaux est moins importante et le poids moyen des animaux au mois de décembre est supérieur à celui du mois de janvier. Les vêlages survenant au $3^{\mathrm{e}}$ trimestre semblent constituer, pour un maintien correct du poids de la femelle, la meilleure période de mise bas.

\section{Evolution pondérale des produits}

L'influence saisonnière sur l'évolution pondérale des produits est importante. L'étude suivante est entreprise sur les naissances observées au C.R.Z. de 1965 à 1972, en travaillant sur les poids relevés tous les mois. Seuls ont été conservés les animaux dont tous les poids sont notés de la naissance à 42 mois pour les mâles et 48 mois pour les femelles. Les résultats concernent des animaux vivant en élevage extensif, se contentant du pâturage naturel.

L'étude porte sur 567 poids de mâles et 927 poids de femelles. Les droites de régression individuelles de l'évolution pondérale par rapport au temps ont été calculées sur les poids suivants : naissance, $6-12-18-24-30-36$ $42-48$ mois. Une analyse de variance a permis de comparer les pentes moyennes de croissance pour chacun des mois de l'année (tableau $n^{\circ} \mathrm{IV}$ )

TABL. $\mathrm{N}^{\circ} \mathrm{IV}$-Pentes moyennes de croissance.

\begin{tabular}{|c|c|c|c|c|}
\hline \multirow{2}{*}{ Mois } & \multicolumn{3}{|c|}{ M a 1 e s } & \multicolumn{2}{c|}{ F e m e 1 1 e } \\
\cline { 2 - 5 } & Nombre & Pente \pm IC & Nombre & Pente \pm IC \\
\hline 1 & 7 & $56,54 \pm 9,76$ & 6 & $37,63 \pm 4,33$ \\
2 & 7 & $52,82 \pm 5,37$ & 16 & $39,79 \pm 3,09$ \\
4 & 8 & $53,17 \pm 5,62$ & 13 & $38,90 \pm 3,11$ \\
5 & 5 & $53,45 \pm 5,24$ & 4 & $38,55 \pm 8,76$ \\
6 & 3 & $60,81 \pm 3,54$ & 11 & $41,83 \pm 5,11$ \\
7 & 8 & $51,85 \pm 6,05$ & 9 & $41,37 \pm 3,32$ \\
8 & 5 & $58,96 \pm 6,13$ & 12 & $42,61 \pm 2,72$ \\
9 & 4 & $56,14 \pm 7,75$ & 9 & $41,81 \pm 4,37$ \\
10 & 6 & $57,51 \pm 3,88$ & 3 & $37,63 \pm 1,54$ \\
11 & 2 & $71,47 \pm 12,77$ & 9 & $41,22 \pm 4,25$ \\
12 & 1 & 42,16 & 1 & 40,30 \\
\hline F & 63 & $1,80 \mathrm{NS}$ & 103 & $0,63 \mathrm{NS}$ \\
\hline
\end{tabular}

IC = Intervalle de confiance.

Aussi bien chez les mâles que chez les femelles, il n'existe pas de différence significative entre les pentes des droites de régression du poids sur l'âge suivant le mois de naissance du produit.

Cependant, en analysant mois par mois, on s'aperçoit que chez les mâles la pente de régres- 
sion du mois de novembre est significativement différente de celles des autres mois $\left(\mathrm{F}=11,33^{* *}\right)$. Il est possible d'attribuer cette différence au faible nombre de données concernant ce mois.

En conséquence, on peut dire que le mois de naissance a peu d'importance sur la croissance future des animaux calculée sur une période longue, soit quatre années qui conduisent environ à l'âge de la commercialisation chez le mâle et celui de la rentrée en reproduction des femelles.

\section{Mortalité des produits en fonction de la période de naissance (5)}

Le taux moyen de mortalité au C.R.Z. de Dara est sur quinze ans de 6,23 p. 100. L'essentiel de la mortalité se produit chez les animaux âgés de moins de deux ans qui représentent 80 p. 100 du nombre total des morts. L'étude a porté sur 486 animaux morts, répartis au cours de l'année. On s'aperçoit que le maximum de mortalité se situe au cours des mois de mai à juillet, soit 45,2 p. $100 \mathrm{du}$ total.

Cependant, si on met cette répartition particulière en parallèle avec celle des naissances, on constate qu'il y a une coïncidence remarquable entre les deux; ce qui permet de conclure que le mois de naissance des produits n'a pratiquement aucune influence sur la mortalité observée de la naissance à 24 mois au C.R.Z. de Dara.

\section{Commodité d'élevage suivant la période de naissance}

L'essentiel est que l'ensemble des naissances soit regroupé à une certaine période de l'année afin que les diverses opérations telles que sevrage, vaccinations, changement d'alimentation... puissent être regroupées sur des lots homogènes. Donc, pour ce facteur, c'est moins la période envisagée que le principe même du regroupement qui est déterminant.

\section{Supplémentation alimentaire $(6,7)$}

Le problème alimentaire est essentiel; dans les conditions sévères du Sénégal Nord, toute amélioration animale passe par une amélioration du milieu, et en particulier des facteurs limitants tels que l'alimentation. Pendant la mauvaise saison, il faut apporter une supplémentation à certaines catégories d'animaux (taureaux, jeunes avant et après le sevrage, femelles des lots de sélection). En particulier, pour les femelles, la question est de savoir s'il vaut mieux supplémenter un jeune en croissance et une vache en lactation plutôt qu'uniquement une vache en gestation. Ici, le facteur période est déterminant : en effet, si la vache met bas en début de saison sèche, sa lactation sera faible et la supplémentation de la femelle et de son produit devra être envisagée. Au contraire, lorsque le vêlage a lieu en début de saison des pluies, la supplémentation de la mère est inutile, la lactation sera meilleure, seule la supplémentation légère donnée au veau compte tenu des faibles capacités laitières de la femelle pourra le conduire valablement au sevrage.

\section{VALEUR DES FACTEURS ETUDIES}

Dans le chapitre précédent, nous avons étudié les facteurs âge au $1^{\text {er }}$ vêlage, intervalles entre les vêlages, mortalité, croissance de la naissance à l'âge adulte, commodité d'élevage qui se sont révélés sans utilité pour chercher à placer une saison de monte.

D'autres facteurs tels la saison naturelle de saillies, l'évolution pondérale de la mère, la supplémentation alimentaire se sont révélés être d'une grande importance.

\section{CHOIX DE LA PERIODE DE MONTE}

La valeur des facteurs étant dégagée, (tableau $n^{\circ}$ V) la période la plus propice à l'installation d'une saison de vêlage est par conséquent en gros le $3^{e}$ trimestre, c'est-à-dire les mois de juillet à septembre. En conséquence, la saison de monte devra se dérouler des mois d'août à novembre. L'application pratique de cette conclusion théorique a été faite au C.R.Z. de Dara depuis 1969.

La première période précise retenue s'est déroulée du $1^{\text {er }}$ septembre au 31 décembre. Ensuite dès 1971, en raison du fait que les saillies étaient rares en décembre, cette saison a été placée du 15 août au 15 décembre. 


\begin{tabular}{|c|c|}
\hline Facteurs étudiés & Période favorable \\
\hline $\begin{array}{c}\text { Répartition naturelle } \\
\text { des naissances }\end{array}$ & Mai à août \\
\hline $\begin{array}{c}\text { Fréquence mensuelle } \\
\text { des saillies }\end{array}$ & $\begin{array}{c}\text { Fin août à } \\
\text { novembre }\end{array}$ \\
\hline Age au 1er vêlage & 0 \\
\hline $\begin{array}{c}\text { Intervalles entre } \\
\text { les vêlages }\end{array}$ & 0 \\
\hline $\begin{array}{c}\text { Evolution pondérale } \\
\text { des vaches }\end{array}$ & $3^{\text {e trimestre }}$ \\
\hline $\begin{array}{c}\text { Evolution pondérale } \\
\text { produits de 0 à 48 mois }\end{array}$ & 0 \\
\hline Mortalité & 0 \\
\hline $\begin{array}{c}\text { Supplémentation } \\
\text { alimentaire }\end{array}$ & en hivernage \\
\hline $\begin{array}{c}\text { Commodité d'élevage } \\
\text { oomm }\end{array}$ & 0 \\
\hline
\end{tabular}

\section{RESULTATS DE L'OPERATION APRES QUATRE ANNEES DE FONCTIONNEMENT}

A vrai dire, depuis 1969 , les conditions pluviométriques sont extrêmement mauvaises au Sénégal, ce qui a eu pour conséquence une diminution catastrophique du disponible fourrager.

Classiquement, l'installation d'une saison de monte induit durant les premières années une diminution du taux global de naissances. Mais en plus, la diminution des possibilités alimen- taires offertes aux animaux provoque un allongement de l'intervalle entre les vêlages et par conséquent une chute du taux de fécondité. La part de l'un ou de l'autre facteur sera difficile à déterminer.

Ceci dit, la répartition des naissances à la suite de la saison de monte va être présentée pour les années 1970 à 1973, de même que quelques données concernant le taux de fécondité.

\section{Répartition des naissances (tableau $n^{0}$ VI)}

En 1970 et en $1971,84,5$ p. 100 des naissances ont été enregistrées de juin à septembre. En 1972,91 p. 100 durant la même période et 83,4 p. 100 en 1973.

Ces résultats amènent à faire plusieurs constatations :

- 15,5 p. 100 des naissances en 1970 et 1971 , 9 p. 100 en 1972 et 16,6 p. 100 en 1973 ont eu lieu en dehors de la période prévue. Ceci tient au fait que le nombre de parcelles du centre étant limité, les animaux mâles et femelles sont relativement proches les uns des autres et les sauts de clôtures par les mâles ne sont pas rares;

- en 1971, 72, 73, on constate que seulement $5,8,8$ et 4,7 p. 100 des naissances ont lieu au mois de septembre et que l'essentiel du taux de naissance se trouve au mois de juin $(38,8-42,0-38,6$ p. 100$)$ ou mieux juin et juillet : $(68,2-65,0-67,7$ p. 100).

Sur le plan pratique, on constate donc l'existence d'une synchronisation alimentaire des animaux nette à la suite de l'abondance de juillet et du début août. En second lieu, il semble possible de réduire la saison de monte à trois mois, du 15 août au 15 novembre.

TABL. $\mathrm{N}^{\mathrm{a}} \mathrm{VI}$-Nombre de raissances et pourcentage

\begin{tabular}{|c|c|c|c|c|c|c|c|c|c|c|c|c|c|}
\hline Années & 1 & 2 & 3 & 4 & 5 & 6 & 7 & 8 & 9 & 10 & 11 & 12 & Total \\
\hline 1970 & - & - & 4 & - & 1 & 27 & 38 & 14 & 21 & 3 & 5 & 5 & 118 \\
& & & 3,3 & & 0,8 & 22,8 & 32,2 & 11,8 & 17,7 & 2,5 & 4,2 & 4,2 & 100 \\
\hline 1971 & 3 & 1 & 0 & 5 & 4 & 33 & 25 & 9 & 5 & 0 & 0 & 0 & 85 \\
& 3,5 & 1,1 & - & 5,8 & 4,7 & 38,8 & 29,4 & 10,5 & 5,8 & - & - & - & 100 \\
\hline 1972 & 0 & 0 & 1 & 1 & 1 & 42 & 23 & 18 & 8 & 1 & 4 & 1 & 100 \\
& 0 & 0 & 1 & 1 & 1 & 42 & 23 & 18 & 8 & 1 & 4 & 1 & 100 \\
\hline 1973 & 2 & 0 & 2 & 4 & 7 & 49 & 37 & 14 & 6 & 2 & 2 & 2 & 127 \\
& 1,6 & - & 1,6 & 3,1 & 5,5 & 38,6 & 29,1 & 11,0 & 4,7 & 1,6 & 1,6 & 1,6 & 100 \\
\hline
\end{tabular}




\section{Variations du taux de naissance}

Le taux de naissance est très variable d'une année à l'autre. Il existe d'une part des variations physiologiques de ce taux proches de 10 p. 100 et d'autre part des modifications de ce taux dues aux différences dans les conditions climatiques. Le tableau $n^{*}$ VII donne une idée de ces variations.

\begin{tabular}{|ll|ll|}
\hline \multicolumn{5}{|c|}{ TABLEAU N $^{\circ}$ VII } \\
\hline 1966 & 71,2 & 1970 & 81,0 \\
1967 & 69,2 & 1971 & 65,0 \\
1968 & 67,7 & 1972 & 67,0 \\
1969 & 72,0 & & \\
\hline
\end{tabular}

Dans ces conditions, il semble difficile de porter un jugement sur l'influence de la saison de monte sur ce taux. Par contre, l'installation de la saison de monte permet de contrôler de façon plus correcte les performances individuelles des femelles.

\section{CONCLUSION}

L'étude des différents facteurs entrant en jeu dans le choix de la saison de monte en milieu tropical sec, montre qu'ils sont de valeur variable pour ce choix. Les facteurs déterminants sont la répartition naturelle des naissances, l'évolution pondérale de la mère, la place de la supplémentation alimentaire, la commodité de la gestion du troupeau. Ils conduisent à préconiser une période de reproduction placée du mois d'août au mois de novembre de chaque année.

Cette période fixée répond au souci de posséder un élevage de rentabilité maximale en utilisant au mieux les conditions naturelles. I1 est évident que certaines modifications pourraient être apportées grâce en particulier à une alimentation améliorée (3) dans le but d'une spéculation commerciale différente, mais il semble qu'il ne soit pas très intéressant de s'éloigner trop du rythme saisonnier actuel.

\section{SUMMARY}

\section{Note on factors allowing the choice of breeding season in the Dara Zootechnical Research Center (Senegal)}

The most appropriate time for breeding season in Gobra zebu cattle starts in the middle of August and ends in November. This conclusion results of studies carried out on natural birth period, monthly frequency of services, age at first calving, intervals between calvings, annual evolution of weights of femals and of their products, mortality and problems of nutrition.

\section{RESUMEN}

Nota sobre los factores determinando el escogimiento de una estación de cubrición en el Centro de investigaciones zootécnicas de Dara (Senegal)

Con el objeto de escoger el periodo más favorable para la cubrición de cebues Gobra en la zona norte de Senegal, se estudiaron la repartición natural de los nacimientos, la frecuencia mensual de las montas, la edad al primer parto, el intervalo entre los partos según el mes de nacimiento del producto, la evolución del peso según la epoca de nacimiento en las hembras y en los productos, la mortalidad, la comodidad de gestión del ganado, la suplementación alimenticia.

Los datos obtenidos muestran que se necesita situar el periodo de cubrición a mediados de agosto hasta el fin de hoviembre en lo concerniendo a la region considerada.

\section{BIBLIOGRAPHIE}

1. DENIS (J.P.). L'intervalle entre les vêlages chez le zébu Gobra. Rev. Elev, Méd. vét. Pays trop., 1971, 24 (4) : 635-647.

2. DENIS (J.P.). Note sur l'âge au premier vêlage. Communication au Congrès international de Zootechnie. Versailles, juillet 1971.
3. DENIS (J. P.). Rapport sur l'influence de l'alimentation sur les performances de reproductrices et de nourrices des femelles Gobra. Dakar, Lab. Nat. Elev. et Rech. vét., mars 1974. (Ronéo), 15 p.

4. DENIS (J. P.) et VALENZA (J.). Comportement pondéral des femelles adultes de race Gobra. 
Comparaison avec les animaux importés Pakistanais et Guzera. Rev. Elev. Méd. vét. Pays trop., 1970,23 (2) : 229-241.

5. DENIS (J. P.) et VALENZA (J.). Etude de la mortalité au C.R.Z. de Dara. Rev. Elev. Méd. vét.
Pays trop., 1972, 25 (3) : 445-454.

6. Rapports annuels C.R.Z. de Dara, 1966-1972.

7. Rapports annuels Laboratoire national de l'Elevage, 1966 à 1972. 